\title{
Enhancement of cooperation through conformity-driven reproductive ability
}

\author{
Han-Xin Yang米 and Lijun Tian讯 \\ ${ }^{1}$ Department of Physics, Fuzhou University, Fuzhou 350116, China and \\ ${ }^{2}$ School of Economics and Management, Fuzhou University, Fuzhou 350116, China
}

(Dated: July 12, 2018)

\begin{abstract}
We propose a conformity-driven reproductive ability in which an individual $i$ is more (less) likely to imitate a neighbor $j$ 's strategy if $j$ 's strategy is majority (minority) in $i$ 's neighborhood. The results on the evolutionary spatial prisoner's dilemma game show that, compared to homogeneous reproductive ability, conformity-driven reproductive ability can greatly enhance cooperation. This finding is robust with respect to different types of network structures (including square lattice and scale-free network) and to different ways of strategy updating (including synchronous and asynchronous strategy updating).
\end{abstract}

PACS numbers: $02.50 . \mathrm{Le}, 87.23 . \mathrm{Kg}, 87.23 . \mathrm{Ge}$

\section{INTRODUCTION}

To understand the emergence of cooperative behavior among selfish individuals, researchers have considered various mechanisms, such as network reciprocity [1, 2], voluntary participation [3, 4], aspiration [5 7], social diversity [8, 9], migration [10 12], chaotic payoff variations [13], extortion [14 16], punishment [17-20], and so on.

The reproductive ability, also is known as the teaching ability or the learning ability, has been extensive studied in the evolutionary games 21 26]. Szolnoki et al proposed an inhomogeneous teaching ability in which the probability that the individual $i$ adopts a randomly chosen neighbor $j$ 's strategy depends on the payoff difference and a two-value pre-factor $\omega$ characterizes the teaching ability of neighbor $j$ [21]. They found that inhomogeneous teaching ability can promote cooperation for the prisoner's dilemma games in lattices [21] and complex networks 22]. Guan et al found that the introduction of the inhomogeneous activity of teaching of individuals can remarkably promote cooperation in spatial public goods games 23]. Szolnoki and Perc defined the teaching ability of a node $i$ as its collective influence which is the product of its reduced degree and the total reduced degree of all $j$ nodes at a hierarchial depth $\ell$ from node $i 24$. It was found that there exists an optimal hierarchical depth for the determination of collective influence that favors cooperation. Chen et al proposed an inhomogeneous learning ability in which the two-value pre-factor $\omega$ characterizes the strength of the individual $i$ 's own learning activity 25]. They found that appropriate intermediate levels of learning activity can promote or sustain cooperation for the prisoner's dilemma games in small-world networks and scale-free networks. Wu et al discovered that cooperation on square lattices is promoted (inhibited) in the case of synchronous (asynchronous) strategy updating, if

\footnotetext{
*Electronic address: yanghanxin001@163.com
}

${ }^{\dagger}$ Electronic address: buaatianlijun@163.com heterogeneous learning ability is considered [26].

In many real-life situations, an individual tends to follow the majority in behavior or opinion within the interaction range. Recently, the consideration of conformity has attracted much attention in the study of evolutionary games. Szolnoki and Perc designated a fraction of population as being driven by conformity rather than payoff maximization [27, 28]. These conformists simply adopt whichever strategy is most common within their interaction range at any given time, regardless of the expected payoff. They showed that an appropriate fraction of conformists within the population introduces an effective surface tension around cooperative clusters and ensures smooth interfaces between different strategy domains.

Motivated by the work of Szolnoki and Perc, we propose a conformity-driven reproductive ability in which the probability that the individual $i$ adopts a randomly chosen neighbor $j$ 's strategy depends on the payoff difference and a pre-factor $\omega_{i j}$ characterizing the popularity of $j$ 's strategy among $i$ 's neighbors. The value of $\omega_{i j}$ is above (below) 0.5 if $j$ 's strategy is majority (minority) in $i$ 's neighborhood. Different from previous works of the teaching ability, the pre-factor in our model is determined not only by $j$ but also by $i$ 's other neighbors.

\section{MODEL}

Our model is described as follows.

Player $x$ can take one of two strategies: cooperation or defection, which are described by

$$
s_{x}=\left(\begin{array}{l}
1 \\
0
\end{array}\right) \text { or }\left(\begin{array}{l}
0 \\
1
\end{array}\right)
$$

respectively. At each time step, each individual plays the prisoner's dilemma game with its nearest neighbors. An individual will punish the neighbors that hold different strategies. The accumulated payoff of player $x$ can thus 
be expressed as

$$
P_{x}=\sum_{y \in \Omega_{x}} s_{x}^{T} M s_{y}
$$

where the sum runs over the nearest neighbor set $\Omega_{x}$ of player $x$ and $M$ is the rescaled payoff matrix given by

$$
M=\left(\begin{array}{ll}
1 & 0 \\
b & 0
\end{array}\right) .
$$

Here the parameter $b(>1)$ denotes the temptation to defect.

Initially, cooperators and defectors are randomly distributed with the equal probability 0.5. Players asynchronously update their strategies in a random sequential order 29 31]. Firstly, an individual $i$ is randomly selected who obtains the payoff $P_{i}$ according to the above equations. Next, individual $i$ chooses one of its nearest neighbors at random, and the chosen neighbor $j$ also acquires its payoff $P_{j}$. Finally, individual $i$ adopts the neighbor $j$ 's strategy with the probability [21]:

$$
W\left(s_{i} \leftarrow s_{j}\right)=\omega_{i j} \frac{1}{1+\exp \left[\left(P_{i}-P_{j}\right) / K\right]},
$$

where $K$ characterizes the noise introduced to permit irrational choices and $\omega_{i j}$ characterizes the ability that $j$ transfers its strategy to $i$.

We define the reproductive ability $\omega_{i j}$ as:

$$
\omega_{i j}=\frac{1}{1+\exp \left[\left(k_{i} / 2-N_{s_{j}}\right) / H\right]},
$$

where $N_{s_{j}}$ is the number of players adopting strategy $s_{j}$ within the interaction range of player $i$ (including $j$ itself), $k_{i}$ is the degree of player $i$, and $H(>0)$ represents the steepness of the function. The more popular is $j$ 's strategy in $i$ 's neighborhood, the higher is the reproductive ability of $j$. For $H=\infty$, the reproductive ability is a constant equaling 0.5 . In this situation, our model is restored to the original homogeneous ability. Conversely, for $H=0$ the reproductive ability becomes steplike so that $i$ refuses to adopt strategy $s_{j}$ if this strategy is minority in $i$ 's neighborhood.

\section{RESULTS}

Following the previous studies [32, 33], we set the noise level to be $K=0.1$. The key quantity for characterizing the cooperative behavior of the system is the fraction of cooperators $\rho_{c}$ in the steady state. In the following simulation, $\rho_{c}$ is obtained by averaging over the last $10^{3}$ Monte Carlo steps (MCS) of the entire $10^{5}$ MCS. Each MCS consists of on average one strategy-updating event for all individuals. Each data is obtained by averaging over 100 different realizations. Unless otherwise specified, all our simulations are performed in a $100 \times 100$ square lattice with the periodic boundary condition.

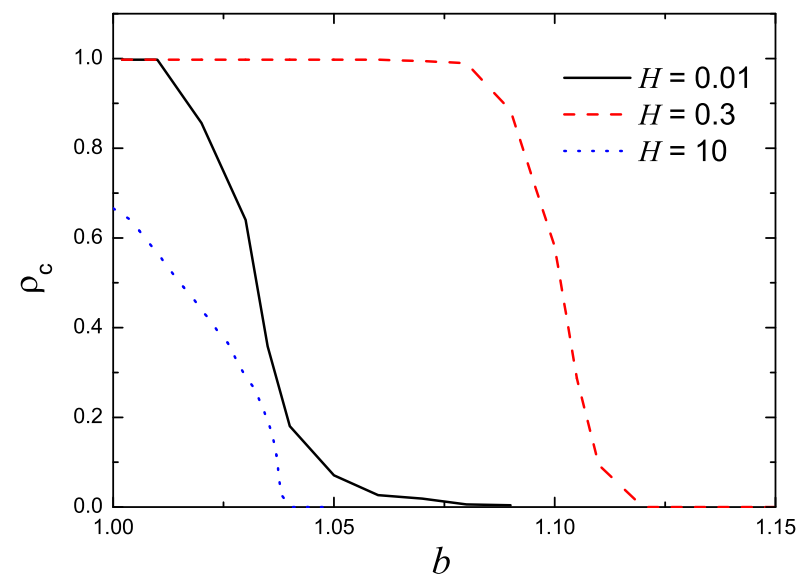

FIG. 1: (Color online) The fraction of cooperators $\rho_{c}$ as a function of the temptation to defect $b$ for different values of the steepness parameter $H$. For each value of $H, \rho_{c}$ decreases to 0 as $b$ increases. For small values of $H$ (e.g., $H=0.01$ or $H=0.3$ ), cooperators can occupy the whole system when $b$ is small. However, for large values of $\alpha$ (e.g., $\alpha=10$ ), full cooperation cannot reach even $b=1$.

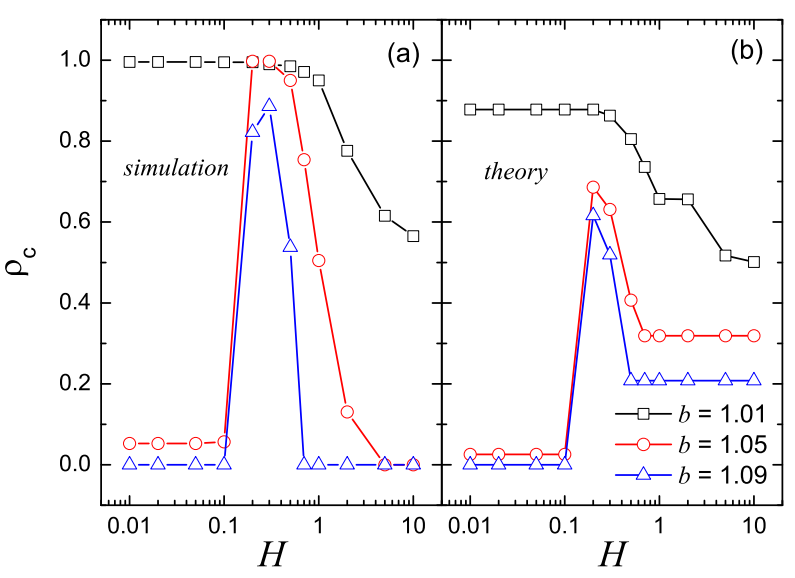

FIG. 2: (Color online) The fraction of cooperators $\rho_{c}$ as a function of the steepness parameter $H$ for different values of the temptation to defect $b$. The results from (a) simulation and (b) theoretical analysis, respectively.

Figure 1 shows the fraction of cooperators $\rho_{c}$ as a function of the temptation to defect $b$ for different values of the steepness parameter $H$. From Fig. 1 one can see that, for each value of $H, \rho_{c}$ decreases to 0 as $H$ increases. For small values of $H$ (e.g., $H=0.01$ or $H=0.3$ ), full cooperation can reach when $b$ is below a threshold value. However, for large values of $H$ (e.g., $H=10$ ), cooperators cannot take over the whole system even $b=1$.

Figure 2 shows the fraction of cooperators $\rho_{c}$ as a function of the steepness parameter $H$ for different values of the temptation to defect $b$. We see that, for relatively small values of $b$ (e.g., $b=1.01), \rho_{c}$ decreases as $H$ increases. However, for larger values of $b$ (e.g., $b=1.05$ 

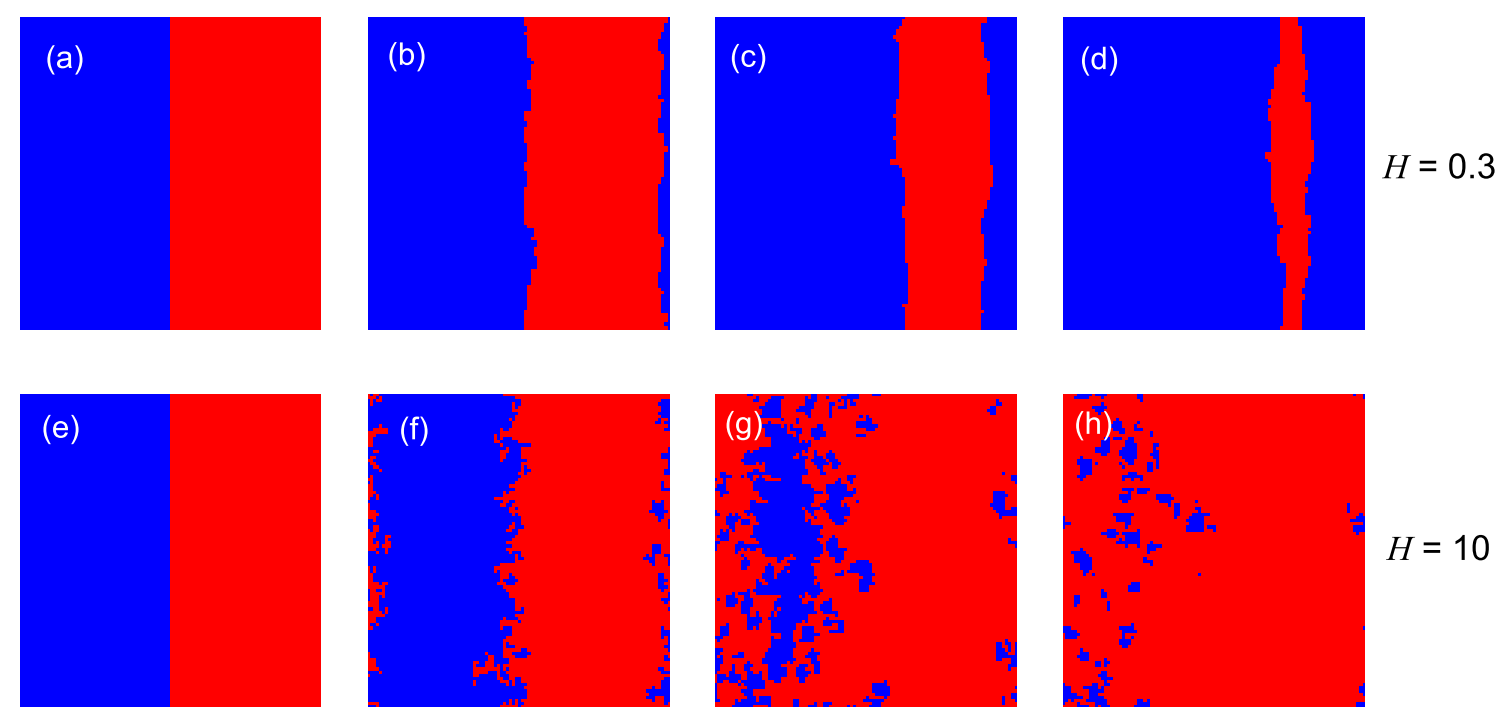

$H=10$

\section{Time}

FIG. 3: (Color online) Snapshots of typical distributions of cooperators (blue) and defectors (red) at different time steps. Initially, we set cooperators (defectors) in the left (right) half of square lattices. The temptation to defect $b=1.05$. The steepness parameter $H$ is $H=0.3$ for (a)-(d) and $H=10$ for (e)-(h). For $H=0.3$, the cooperator cluster continually expands and the boundary between the two competing clusters keeps smooth during the whole evolution. For $H=10$, the defector cluster continually expands and the boundary becomes littery as time evolves.

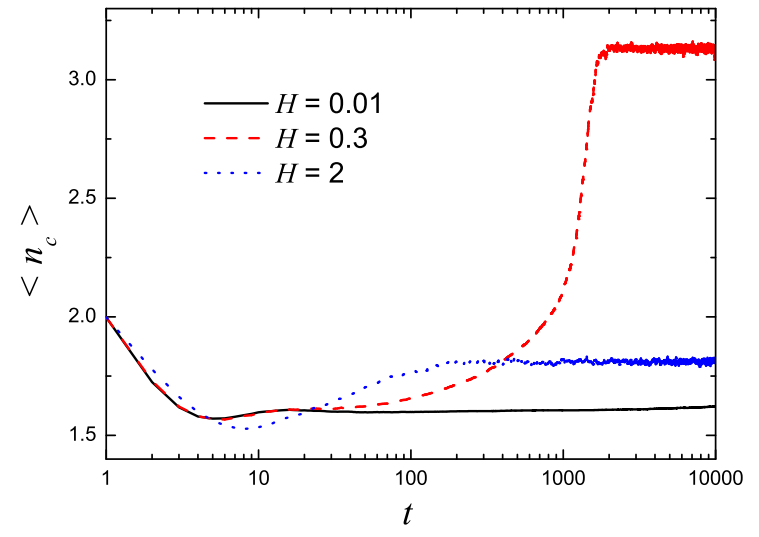

FIG. 4: (Color online) The time evolution of the average number of cooperative neighbors $\left\langle n_{c}\right\rangle$ for players along the interfaces separating domains of cooperators and defectors. We define a player along the the interface as the one who has at least one neighbor with the opposite strategy. The temptation to defect $b=1.05$. For each value of the steepness parameter $H,\left\langle n_{c}\right\rangle$ firstly decreases and then increases as time evolves.

or 1.09), there exists an optimal value of $H$ (about 0.2 ) leading to the highest cooperation level. The dependence of $\rho_{c}$ on $H$ can be qualitatively predicted analytically through a pair-approximation analysis [34, 35], the results of which are shown in Fig. 2(b).
To intuitively understand why the moderate value of $H$ that can best enhance cooperation, we plot spatial strategy distributions as time evolves for different values of $H$ when the temptation to defect $b=1.05$. Initially we set a giant cooperator (defector) cluster in the left (right) half of square lattices. From Figs. 2(a)-(d), one can see that for the moderate value of $H$ (e.g., $H=0.3)$, the cooperator cluster continually expands while the defector cluster gradually shrinks. Note that for $H=0.3$, the boundary between the two competing clusters remains smooth during the whole evolution. However, for the large value of $H$ (e.g., $H=10$ ), the defector cluster gradually invade the cooperator cluster and the original one big cooperator cluster is divided into some small clusters [see Figs. 2(e)(h)]. For $H=10$, the interfaces separating domains of cooperators and defectors become littery. As pointed out in Ref. [36, 37], noisy borders are beneficial for defectors, while straight domain walls help cooperators to spread. For the very small value of $H$ (e.g., $H=0.01$ ), the cooperator and defector clusters keep almost unchanged (the results are not shown here).

Next, we study the average number of cooperative neighbors $\left\langle n_{c}\right\rangle$ for players along the interfaces separating domains of cooperators and defectors. A player is along the interface if it has at least one neighbor with the opposite strategy. Figure 4 shows the time evolution of $\left\langle n_{c}\right\rangle$ for different values of the steepness parameter $H$ when the temptation to defect $b=1.05$. One can see that 

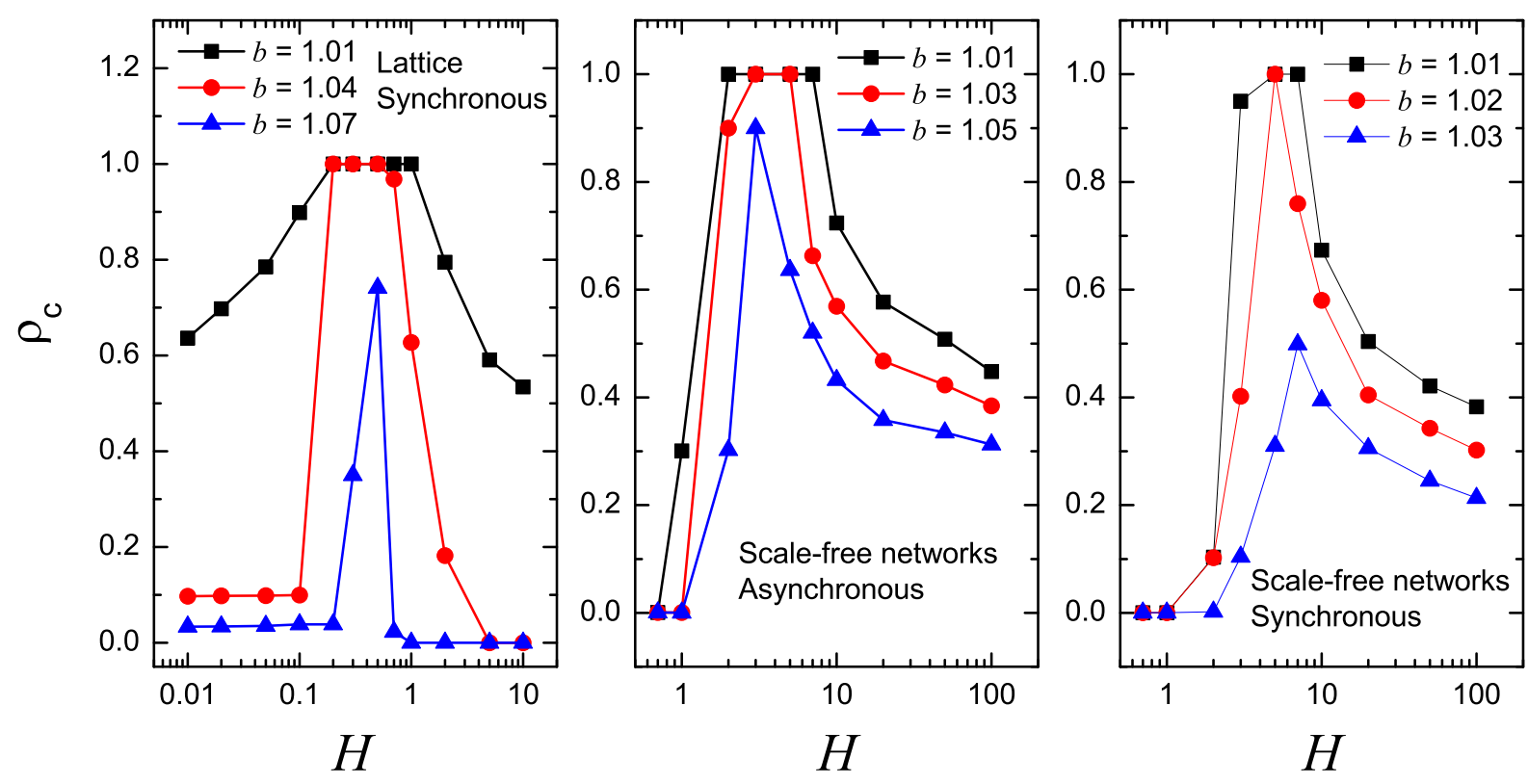

FIG. 5: (Color online) The fraction of cooperators $\rho_{c}$ as a function of the steepness parameter $H$ for different values of the temptation to defect $b$ under different types of networks and different kinds of updating rules. Left panel: for square lattices and synchronous updating rule. Middle panel: for scale-free networks and asynchronous updating rule. Right panel: for scale-free networks and synchronous updating rule. The network size is set to be 10000 and the average degree of the network is 4 . Note that for scale-free networks, we use degree-normalized payoffs.

initially $\left\langle n_{c}\right\rangle$ decreases from 2 to about 1.6 , and then increases to a stable value. For the small value of $H$ (e.g., $H=0.01)$ and the large value of $H$ (e.g., $H=2$ ), the final value of $\left\langle n_{c}\right\rangle$ is below 2 . However, for the moderate value of $H$ (e.g., $H=0.3$ ), $\left\langle n_{c}\right\rangle$ finally reaches 3.1 . Once the value of $\left\langle n_{c}\right\rangle$ exceeds 2 , cooperation becomes the majority strategy in a player's neighborhood. In this case, the conformity-driven reproductive ability is beneficial for the expansion of cooperator clusters.

In all the above studies, we use square lattices and asynchronous strategy updating. In fact, our finding that the moderate value of the steepness parameter $H$ can best promote cooperation is robust with respect to different kinds of network structures and different ways of strategy updating. Since square lattice is homogeneous interaction networks, it is interesting for us to consider heterogeneous interaction networks. We use the famous Barabási-Albert scale-free networks to construct heterogeneous interaction 38]. In the asynchronous updating rule, at each time only a randomly selected player is allowed update its strategy. While in synchronous updating rule, at each time all players update their strategies. We consider three cases: square lattices with synchronous strategy updating, scale-free networks with asynchronous strategy updating and scale-free networks with synchronous strategy updating. From Fig. 5. one can see that for all these cases, the cooperation level reaches the highest at the moderate value of $H$ when the temptation to defect $b$ is fixed.

\section{CONCLUSIONS AND DISCUSSIONS}

To summarize, we have proposed a conformity-driven reproductive ability in which the probability that a player $i$ adopts a neighbor $j$ 's strategy depends on their payoff difference and a pre-factor $\omega_{i j}$ characterizing the popularity of $j$ 's strategy among $i$ 's neighbors. The value of $\omega_{i j}$ increases with the number of $i$ 's neighbors holding the same strategy with $j$. Both numerical and theoretical results show that, the cooperation level of the spatial prisoner's dilemma game can be greatly enhanced by moderately increasing the teaching ability of the neighbor with the majority strategy in the local community. In the case of the conformity-driven reproductive ability, the borders of cooperator clusters become smooth, thus cooperators along the borders can get more help to resist the invasion of defectors. Note that the concept of conformity is widely existent in opinion dynamics [39, 40]. We hope our work can attract more interest in the study of the heterogeneous reproductive ability based on the opinion dynamics.

\section{Acknowledgments}

This work was supported by the National Natural Science Foundation of China (Grants Nos. 61403083, 71301028 and 71671044), and Excellent Youth Science Foundation of Fujian Province (Grant No. 2016J06017). 
[1] Perc M, Gómez-Gardeñes J, Szolnoki A, Floría L M, Moreno Y. J R Soc Interface 2013;10:20120997.

[2] Wang Z, Wang L, Szolnoki A, Perc M. Eur. Phys. J. B 2015;88:124.

[3] Szabó G, Hauert C. Phys Rev Lett 2002;89:118101.

[4] Wu ZX, Xu XJ, Chen Y, Wang YH. Phys Rev E 2005;71:037103.

[5] Chen X, Wang L. Phys Rev E 2008;77:017103.

[6] Liu Y, Chen X, Wang L, Li B, Zhang W, Wang H. EPL 2011;94:60002.

[7] Amaral MA, Wardil L, Perc M, da Silva JKL. Phys Rev E 2016;94:032317.

[8] Perc M, Szolnoki A. New J Phys 2008;10:043036.

[9] Yang HX, Wang WX, Wu ZX, Lai YC, Wang BH. Phys Rev E 2009;79:05610.

[10] Helbing D, Yu W. Proc Natl Acad Sci USA 2008;106:3680.

[11] Meloni S, Buscarino A, Fortuna L, Frasca M, GómezGardeñes J, Latora V, Moreno Y. Phys Rev E 2009;79:067101.

[12] Chen X, Szolnoki A, Perc M. Phys Rev E 2012;86:036101.

[13] Perc M. EPL 2006;75:841.

[14] Szolnoki A, Perc M. Phys Rev E 2014;89:022804.

[15] Hao D, Rong Z, Zhou T. Phys Rev E 2015;91:052803.

[16] Rong Z, Wu ZX, Hao D, Chen MZQ, Zhou T. New J Phys 2015;17:033032.

[17] Szolnoki A, Szabo G, Czako L. Phys Rev E
2011;84:046106

[18] Szolnoki A, Perc M. Phys Rev X 2013;3:041021.

[19] Chen X, Szolnoki A, Perc M. Phys Rev E 2015;92:012819.

[20] Chen X, Sasaki T, Perc M. Sci Rep 2015;5:17050.

[21] Szolnoki A, Perc M. EPL 2007;77:30004.

[22] Szolnoki A, Perc M, Szabó G. Eur Phys J B 2008;61:505.

[23] Guan JY, Wu ZX, Wang YH. Phys Rev E 2007;76:056101.

[24] Chen X, Fu F, Wang L. Int J Mod Phys C 2008;19:1377.

[25] Wu ZX, Rong Z, Chen MZQ. EPL 2015;110:30002.

[26] Szolnoki A, Perc M. EPL 2016;113:58004.

[27] Szolnoki A, Perc M. J R Soc Interface 2015;12:20141299.

[28] Szolnoki A, Perc M. Sci Rep 2016;6:23633.

[29] Wang Z, Szolnoki A, Perc M. Sci Rep 2013;3:2470.

[30] Wang Z, Szolnoki A, Perc M. J Theor Biol 2014;349:50.

[31] Wang Z, Wang L, Perc M. Phys Rev E 2014;89:052813.

[32] Wu ZX, Rong Z. Phys Rev E 2014;90:062102.

[33] Yang HX, Wu ZX, Rong Z, Lai YC. Phys Rev E 2015;91:022121.

[34] Szabó G, Tőke C. Phys Rev E 1998;58:69.

[35] Hauert C. Szabó G. Am J Phys 2005;73:405.

[36] Szolnoki A, Wang Z, Perc M. Sci Rep 2012;2:576.

[37] Perc M, Szolnoki A. New J Phys 2012;14:043013.

[38] Barabási, Albert R. Science 1999;286:509.

[39] Krapivsky PL, Redner S. Phys Rev Lett 2003;90:238701.

[40] Wu ZX, Holme P. Phys Rev E 2009;81:011133. 